\title{
Distribution of fundus autofluorescence with a scanning laser ophthalmoscope
}

\author{
A von Rückmann, F W Fitzke, A C Bird
}

\begin{abstract}
Background-Variation of fluorescence derived from lipofuscin in the retinal pigment epithelium has been recorded with age and in retinal diseases. Studies have been based largely on in vitro observations on eye bank eyes which has placed severe limitations on the data available.

Methods-A technique is described whereby in vivo imaging of autofluorescence of the fundus was achieved using a scanning laser ophthalmoscope.

Results-The optical characteristics, distribution, and variation with disease imply that the fluorescence is derived from lipofuscin in the pigment epithelium. Autofluorescence is shown to be abnormally high in certain inherited diseases, and low in the presence of retinal atrophy.

Conclusion-This technique may be useful both in clinical practice and research. It may allow the detection of the abnormal phenotype in genetically determined disease at a time when other techniques may not. Longitudinal studies of age related macular disease would permit correlation between changes in the pigment epithelium and Bruch's membrane to be established.
\end{abstract}

(Brf Ophthalmol 1995; 79: 407-412)

In common with all metabolically active but non-dividing cells accumulation of lipofuscin occurs in the retinal pigment epithelium with age. ${ }^{1-14}$ The presence of lipofuscin is thought to reflect the metabolic activity which is determined largely by the rate of turnover of photoreceptor outer segments. It is reported that pigment epithelial autofluorescence increases up to the age of 20 years, remains relatively constant for a further 10-20 years, and then increases once more. ${ }^{15}$ There is good evidence that the pigment epithelium discharges cytoplasmic contents into the inner portion of Bruch's membrane in order to achieve limited cellular renewal, and that this process is a main contributor to age related change in Bruch's membrane. ${ }^{16-19}$ Thus, a relation between the accumulation of debris in both the retinal pigment epithelium and Bruch's membrane might be expected. Abnormally high levels of lipofuscin have been shown in the retinal pigment epithelium in some inherited retinal diseases. ${ }^{20-26}$

It is evident that in vivo recording of pigment epithelial autofluorescence as an index of lipofuscin accumulation may give important clues as to the pathogenesis and progress of a number of retinal diseases. However, our understanding of lipofuscin levels in disease states is based largely on histopathological observations of a small number of eyes. Few clinical studies exist, but these suggest considerable potential. The most recent involved spectrophotometric measurement of autofluorescence intensity at $7^{\circ}$ temporal to the fovea and in the fovea. ${ }^{27}$ Fundus autofluorescence which may contribute to imaging during fluorescein angiography with a scanning laser ophthalmoscope (SLO) ${ }^{28}$ is very low in magnitude. Based on histopathological observations, the spatial distribution of lipofuscin in disease states may be complex. Therefore, accurate imaging of the distribution of autofluorescence in the pigment epithelium, and studies of the dynamics of accumulation would be necessary to clarify the relation between the pigment epithelial autofluorescence and retinal disease. With the SLO we describe here and appropriate excitation light and barrier filters this objective can be achieved. The instrument images the retina in three dimensions by using confocal optics, which allows an object to be imaged in a specific depth plane. ${ }^{29}$ We have studied a number of patients with a variety of fundus changes and have shown variation of autofluorescence between cases and within the same fundus.

Subjects and methods

The confocal scanning laser ophthalmoscope (cSLO) was a prototype SM 30-4024 donated by Zeiss (Zeiss, Oberkochen, Germany). The argon laser blue line ( $488 \mathrm{~nm} ; 250 \mu \mathrm{W})$ was used for illumination, and to record autofluorescence a broad band pass barrier filter with a short wavelength cut off at $521 \mathrm{~nm}$ was

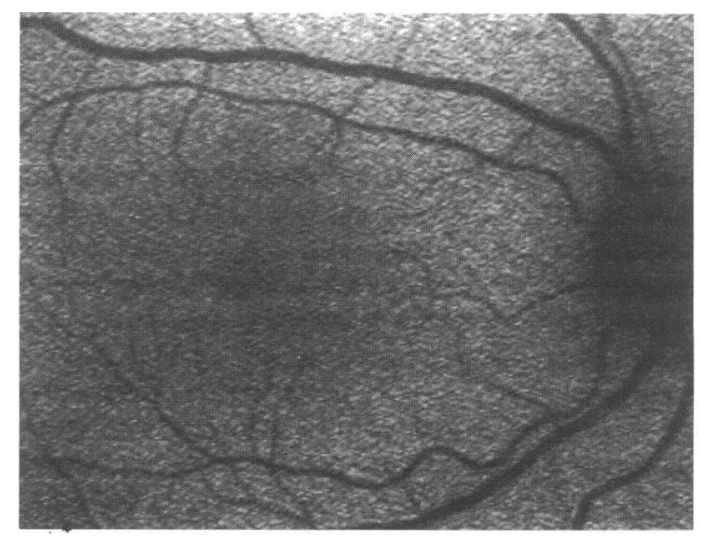

Figure 1 A 35-year-old woman with normal retina. Visual acuity was $6 / 6$ in both eyes. Fluorescence imaging showed diffuse fundus fluorescence with decreased intensity at the fovea and blood vessels and optic disc shown as negative shadows. 


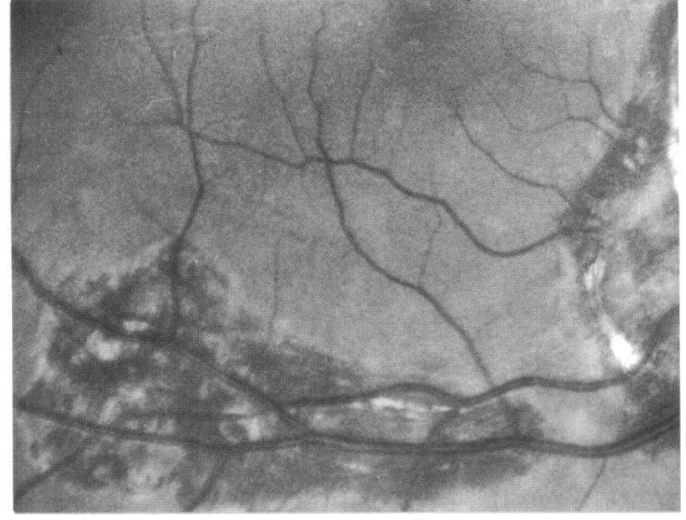

Fig $2 A$

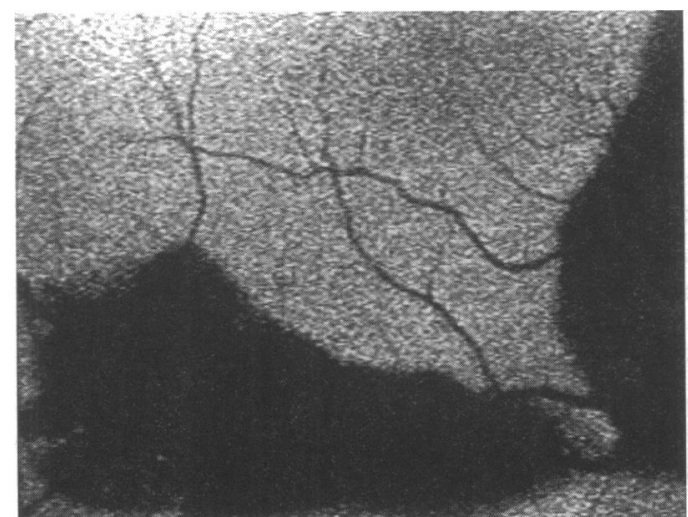

Fig $2 B$

Figure 2 A 53-year-old woman with helicoid degeneration. Right eye visual acuity was $6 / 9$ and there was well defined profound atrophy of the outer retina, and hyperpigmentation following the retinal vessel $(A)$. Fluorescence imaging showed hypofluorescence which corresponded with the atrophy $(B)$.

inserted in front of the detector. The cSLO images were recorded at standard video scanning rates on SVHS video tape and digitised at $256 \times 256$ resolution using a Wild Vision V-10 frame grabber (Wild Vision, Tyne and Wear, UK) with an Acorn Archimedes computer (Acorn Computers, Cambridge, UK). The axial resolution of the cSLO was determined in a model eye using a method that has been previously described. ${ }^{29}$

A total of 369 eyes from 186 patients with retinal diseases and 60 eyes of 30 normal subjects were examined. The age range of the patients was 6 to 75 (mean 54) years and normal subjects 6 to 76 (mean 49) years. The pupil was dilated with phenylephrine $2 \cdot 5 \%$ and tropicamide $1 \%$ before imaging. The $40^{\circ}$ field of view mode was used to produce images of the fundus and of the distribution of retinal autofluorescence. In practice, when using confocal aperture 1 the image quality suffered from insufficient light levels even with maximum illumination, but the signal was good using the confocal aperture 3 in both the $20^{\circ}$ and $40^{\circ}$ of view mode. The ametropic corrector was used to correct for refractive error and for focusing on the structure of interest, following which images were recorded. The barrier filter was then moved into place in front of the detector in the cSLO and a further series of images were recorded. Fundus images were recorded using a video tape recorder

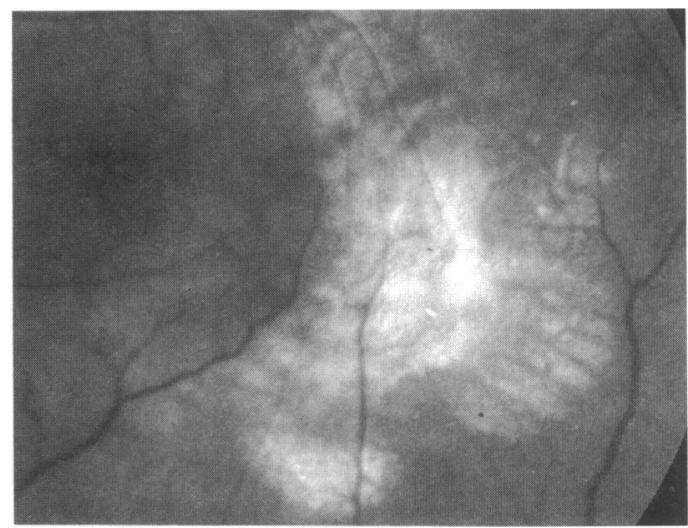

Fig $3 A$

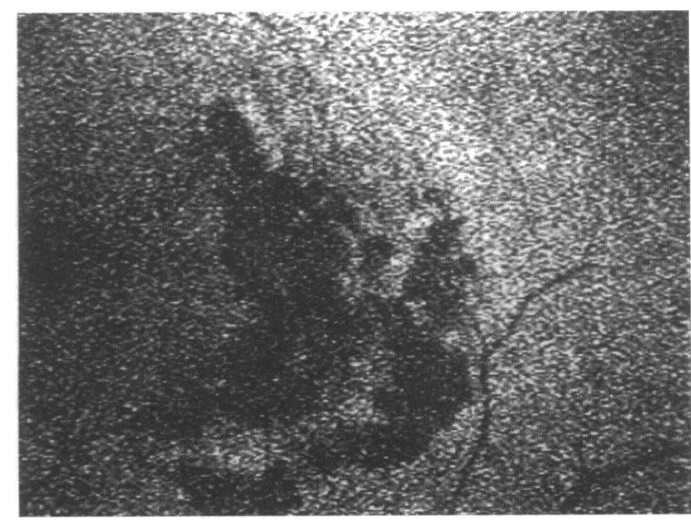

Fig $3 B$

Figure 3 A 72-year-old man with age related macular disease. Left eye visual acuity was $1 / 60$ and there was well defined profound atrophy of the outer retina temporal to the fovea due to a rip of the retinal pigment epithelium $(A)$. Fluorescence imaging showed hypofluorescence which corresponded with the atrophy (B).

(Panasonic AG-7330, Matsushita Electric Industrial Co, Osaka, Japan). Thirty two individual images digitised at six frames per second were aligned and averaged to reduce noise. The selection of six frames per second was determined by the speed limitations of transfer of each digital image to mass storage. Images were aligned by flickering between the first image and each of the subsequent images in the series while moving these using a computer mouse to minimise apparent motion using a method previously described. ${ }^{30}$ Landmarks such as vessels and other fundus features were used for alignment. The reflectance images were compared with autofluorescence images and with colour slides obtained using a fundus camera.

\section{Results}

In the model eye the axial resolution of the cSLO was measured and confirmed to be under $300 \mu \mathrm{ms}$ for the 40 of view and confocal aperture 3. Focusing on the retinal surface, and knowing the axial resolution of the cSLO, ensured that the autofluorescence recorded after putting the barrier filter in place arose from the ocular fundus.

We found that computer averaging over 32 frames produced more detailed images of the spatial distribution of fundus autofluorescence and effectively reduced noise in the images. This is important especially in imaging mild or 


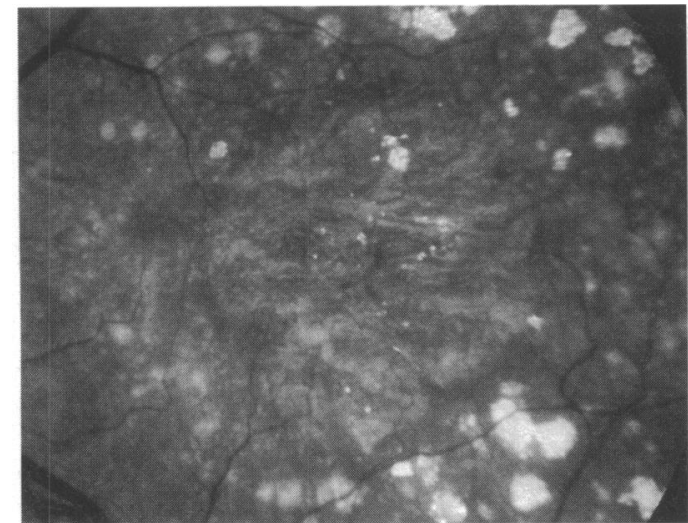

Fig $4 A$

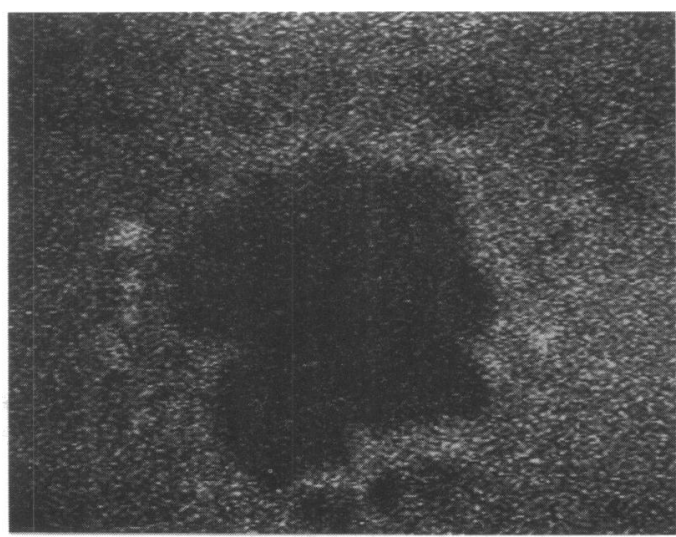

Fig $4 B$

Figure 4 A 67-year-old woman with age related macular disease. Left eye visual acuity was $6 / 60$ and there was geographical atrophy of the outer retina at the fovea and crystalline drusen in the peripheral macula $(A)$.

Fluorescence imaging showed hypofluorescence which corresponded with the atrophy but the drusen do not fluoresce (B).

early disease states where dynamic video recording did not document small alterations of fundus autofluorescence.

In the normal fundi there was diffuse autofluorescence in subjects over the age of 15 years, with the retinal blood vessels and optic disc shown as negative shadows (Fig 1). Without the barrier filter in place the brightest reflecting structure was the optic nerve head. With the barrier filter in place this became the darkest structure suggesting that no significant leakage through the barrier filter contributed to the hyperfluorescence seen in these images. At the fovea there was local hypofluorescence which was probably due in part to absorption of short wavelength light by luteal pigment. The topographical distribution of fundus autofluorescence in normal eyes demonstrated a consistent pattern with a reduction of intensity at the fovea.

This pattern was altered in disease. In subjects in whom there were areas of long standing retinal atrophy, absent autofluorescence corresponded well spatially with the atrophy but was present in the adjacent regions where there was surviving retina (Figs 2-5). Although in one case (Fig 2) there was hyperpigmentation at the site of the lesion, the distribution and intensity of hypofluorescence did not correspond precisely with that of the pigmentation.

In the presence of disease in which there is

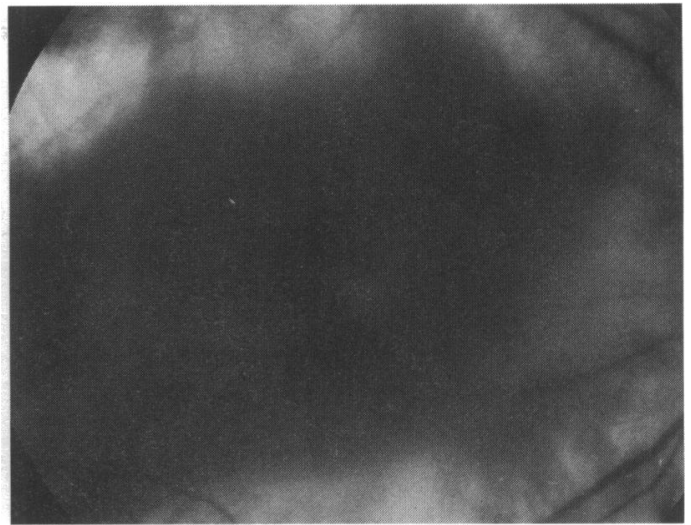

Fig $5 A$

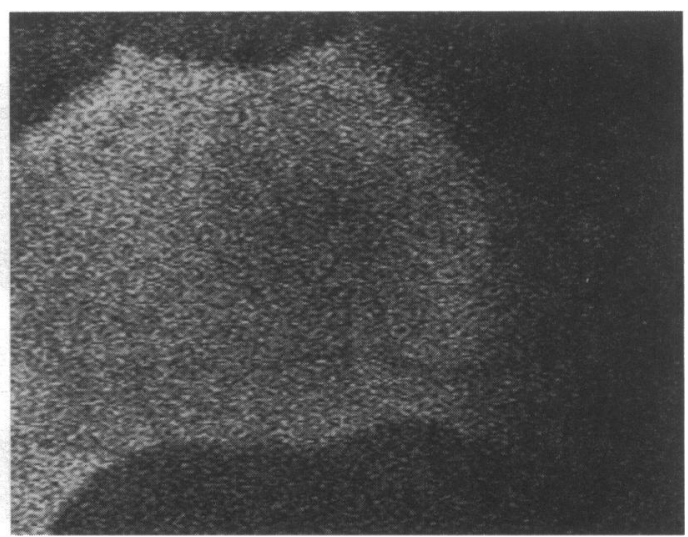

Fig $5 B$

Figure 5 A 48-year-old woman with autosomal dominant retinitis pigmentosa with well defined profound atrophy of the outer retina, retinal pigment epithelium and inner chord except at the macula $(A)$. Right eye visual acuity was $6 / 6$. Fluorescence imaging showed hypofluorescence at the site of atrophy and normal fluorescence corresponding with the viable retina $(B)$.

believed to be excessive accumulation of lipofuscin in the retinal pigment epithelium, there was diffuse and intense hyperfluorescence which was far in excess of that seen with aging (Figs 6-8). In inherited disease the white deposits seen on ophthalmoscopy were relatively more fluorescent than the background, although the gain had to be attenuated for this to be apparent in two cases, one with adult vitelliform macular degeneration and one with pattern dystrophy (Figs 7,8 ). Drusen as a manifestation of age related disease were not consistently fluorescent (Fig 4).

\section{Discussion}

It is most likely that the fundus autofluorescence imaged with the cSLO in our study is derived from lipofuscin in the retinal pigment epithelium. The major fluorescent chromophore in the ocular fundus is lipofuscin, and the fluorescence characteristics and distribution of lipofuscin would explain the images obtained. When exposed to a blue green excitation light at 450-500 $\mathrm{nm}$, lipofuscin fractions from all age groups exhibit a strong broad band of fluorescence emission with a peak between 550 and $600 \mathrm{~nm} .{ }^{714}$ The wavelength $(488 \mathrm{~nm})$ of the argon laser and the barrier filter used in our study are appropriate for detecting the autofluorescence of 


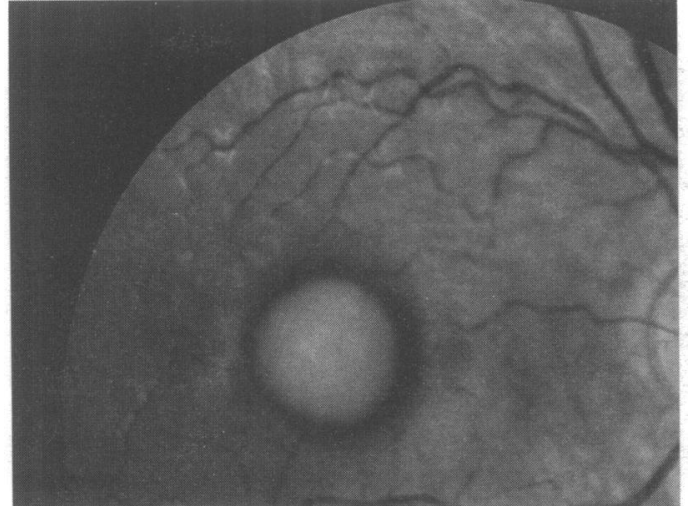

Fig $6 A$

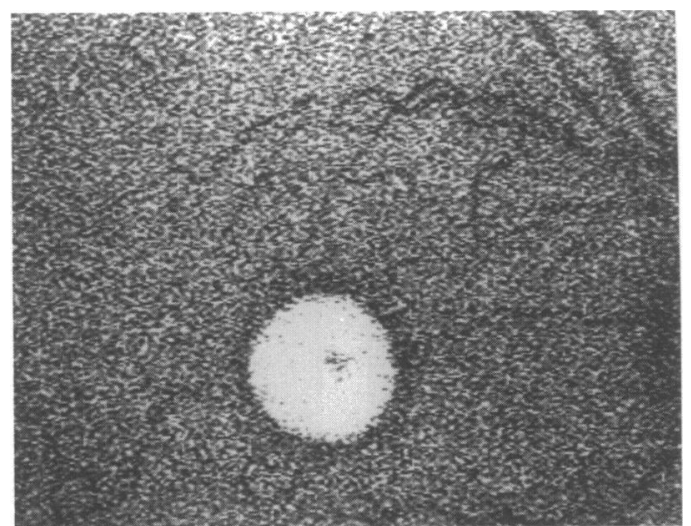

Fig $6 B$

Figure 6 A 6-year-old boy with Best's disease with a well defined white deposit at the level of the retinal pigment epithelium centrally $(A)$. Right eye visual acuity was 6/6. Fluorescence imaging showed hyperfluorescence which corresponded with the deposit (B). The surrounding fundus was also more hyperfluorescent than would be expected at this age.

lipofuscin. Other autofluorescent compounds in the pigment epithelium can probably be discounted as making a significant contribution. Melanin granules are autofluorescent but they exhibit weak emission compared with lipofuscin when excited with short wavelength light. ${ }^{14}$ Moreover, pigmented retinal scars appeared hypofluorescent compared with the surrounding clinically unaffected areas. This suggests that the autofluorescence exhibited by high concentrations of melanin is weak when compared with that of lipofuscin under the illumination conditions used in our study. Furthermore, the distribution of fluorescence from pigmented scars did not correspond precisely with that of melanin implying that absorption of light was not important. The RPE melanin content in normal eyes decreases from the equator to the posterior pole, with a consistent peak of RPE melanin content at the macula while for lipofuscin the reverse is the case. ${ }^{14}$ The fluorescence recorded corresponded with that of lipofuscin rather than of melanin. When excited with short wavelength, vitamin A also emits a weak fluorescence compared with lipofuscin fluorescence with a peak at $495 \mathrm{~nm} .{ }^{7}$ The barrier filter used in the present study eliminates much of the fluorescence emitted by vitamin A. Thus, it seems unlikely that sites of vitamin A storage contribute significantly to the retinal fluorescence imaged in this study.

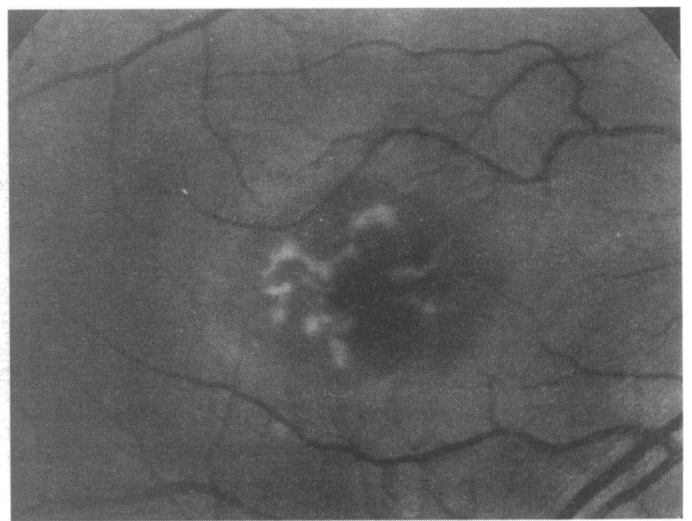

Fig $7 A$

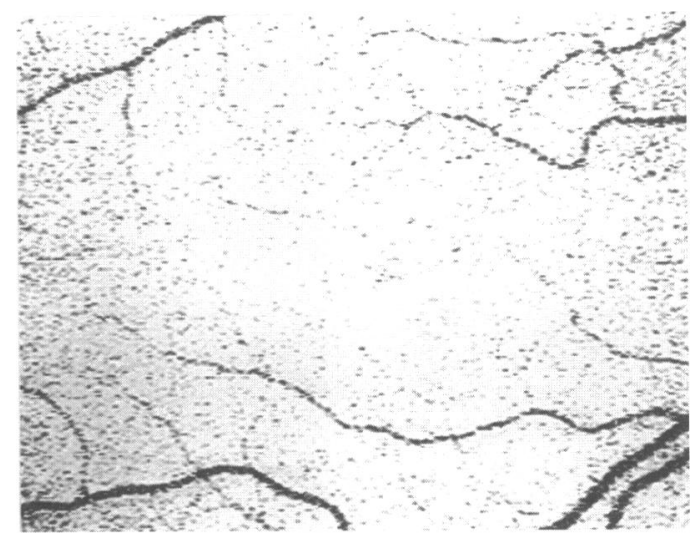

Fig $7 B$

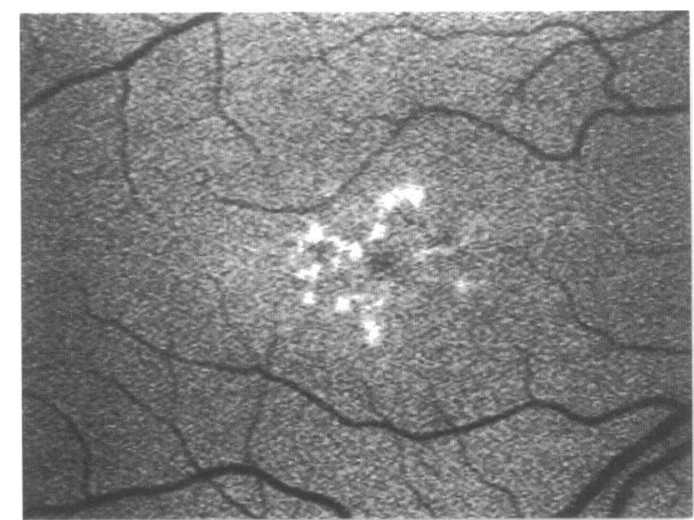

Fig $7 C$

Figure 7 A 28-year-old woman with pattern dystrophy characterised by linear pale deposits at the level of the retinal pigment epithelium (A). Right eye visual acuity was 6/24. Fluorescence imaging showed diffuse

hyperfluorescence of the posterior pole $(B)$. When the

fluorescence was attenuated by 0.7 log unit the pale lesions were relatively more fluorescent than the surrounding fundus (C).

That the source of the autofluorescence is in the retinal pigment epithelium is supported by the distribution of fluorescence both in normal subjects and in disease. The relative hypofluorescence of the optic nerve head implies that the light reaching the detector is not a consequence of mismatching of the filters or fluorescence generated in the ocular media such as may occur in the lens. If there were a mismatch of the filter generating the signal, then the highly reflectant optic nerve head would appear brighter than the surrounding retina. The confocal nature of the optics excludes any potential lens contribution. Furthermore, the confocal optics of the cSLO 


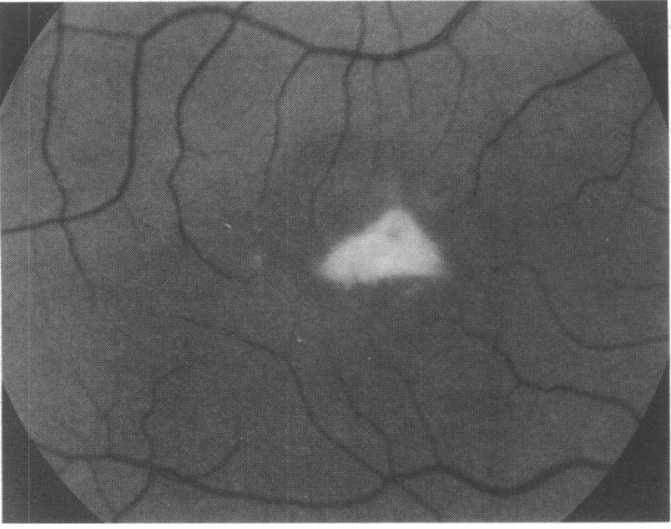

Fig $8 A$

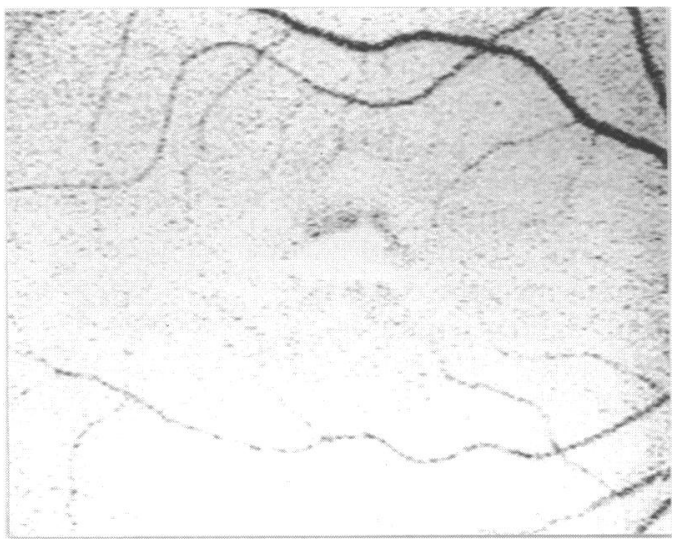

Fig $8 B$

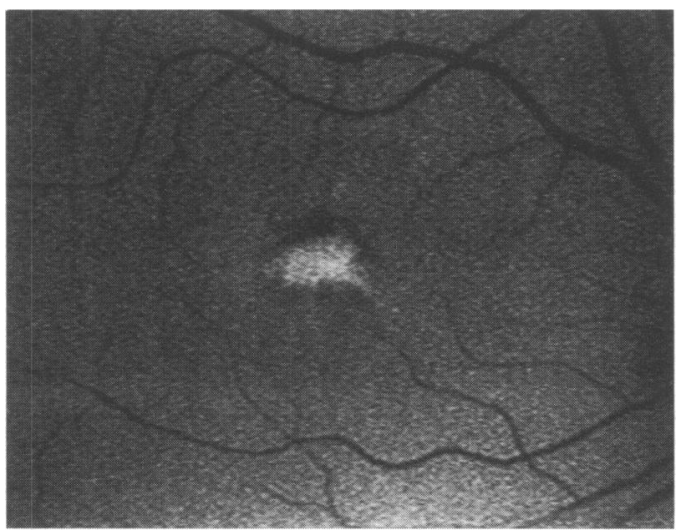

Fig $8 C$

Figure 8 A 39-year-old woman with adult vitelliform macular dystrophy causing reduction of visual acuity to 6/60. There was a well defined white deposit at the level of the retinal pigment epithelium $(A)$. Fluorescence imaging showed diffuse hyperfluorescence $(B)$ and attenuation of the fluorescence by 0.7 log units revealed that the deposit was relatively more hyperfluorescent than the surrounding retina $(C)$.

ensured that the reflectance and fluorescence measurements for one setting of the ametropic corrector is derived from the same optical section ${ }^{29}$ which would effectively exclude autofluorescence from lens and anterior media.

The distribution and intensity of fluorescence observed corresponds with current concepts of the distribution of lipofuscin in disease derived from in vitro fluorescence microscopy of donor eyes ${ }^{20-26}$ and from the limited in vivo studies which exist. ${ }^{27} 31$ It is believed that lipofuscin accumulation in the pigment epithelium reflects the level of metabolic activity which is largely determined by the quantity of photoreceptor outer segment renewal. There is evidence of constant degradation of lipofuscin so that its presence would depend upon continuing metabolic demand. ${ }^{2}$ If there is photoreceptor death, progressive loss of lipofuscin in the retinal pigment epithelium would occur. Thus the hypofluorescence in areas of retinal atrophy as shown in our cases would be expected. This is shown in Figure 3, and would explain the reports of variation in fluorescence using nonimaging techniques. ${ }^{27}$

That there would be hyperfluorescence in certain inherited disorders would also be predictable. In both Best's disease and pattern dystrophies it has been shown that there is diffuse accumulation of lipofuscin in the pigment epithelium ${ }^{20-26}$ which would account for the marked diffuse and focal hyperfluorescence seen in Figures 6-8. The hyperfluorescence may be a consequence of increased rates of outer segment renewal, ${ }^{32} 33$ or defective degradation of phagosomal material by lysosomal enzymes. ${ }^{2}$

The usefulness of this technique to research and clinical management will become evident with time. Histopathological studies have shown that accumulation of lipofuscin in the retinal pigment epithelium is an early feature of some disorders at a time when ophthalmoscopic changes are absent, and before functional loss occurs or at least would be hard to identify. ${ }^{25}$ In some patients shown to have mutation in the peripherin gene causing pattern dystrophy, increased autofluorescence was first phenotypic expression of a retinal dystrophy and thus permit early diagnosis. Thus the technique allows more complete documentation of the distribution of the abnormal gene within the family than would be possible by clinical examination alone. The documentation of autofluorescence may also be valuable in defining the disease state. This is evident particularly in pattern dystrophy in which there may be only small visible pale deposits at the level of Bruch's membrane, ${ }^{33}$ making the distinction from early age related change difficult. If it can be shown that there is diffuse and intense pigment epithelial autofluorescence the diagnosis of inherited disease can be supported. Accumulation of lipofuscin is also reported to occur in some cases of retinitis pigmentosa but not others. ${ }^{34} 35$ The dependence on histopathological studies places severe limitations on the data available, and precludes longitudinal studies. For example it is not known if such an accumulation occurs in all patients with retinitis pigmentosa at some stage of evolution of disease. If this were not the case, imaging of fundus autofluorescence might serve to distinguish one form of retinitis pigmentosa from another and give an indirect indication of progression of disease. Type I (diffuse) retinitis pigmentosa, in which the photoreceptors have a near normal compliment of rhodopsin after loss of scotopic sensitivity, may be different from type II (regional) disease in which there is outer segment loss early in disease. ${ }^{36-39}$ Finally, the nature of the material within the visible pale deposits at the level of the retinal pigment epithelium and Bruch's 
membrane seen in various disease states is incompletely documented. For example, it is not known if the chemical constitution of such deposits in Best's disease, fundus flavimaculatus, pattern dystrophies, and age related macular degeneration is similar. It is evident that drusen may not be intensely autofluorescent but the significance of this observation to disease has yet to be established.

Recording the intensity, spatial distribution and changes in fluorescence over time may add a great deal of information to the longitudinal studies of retinal diseases. This may be particularly important in age related macular disease in that it would give insight into the relation between accumulation of material in the pigment epithelium and Bruch's membrane.

Refinements to the technique are required if accurate quantitation is to be achieved. Compensation would be necessary for the influence of nuclear sclerosis in the elderly, which would cause selective absorption of short wavelength light, and confound measurement. Variation in the melanin content of both the choroid and pigment epithelium may also influence the results. Neither of these appear to have modified our recording of spatial distribution of fluorescence, and would not account for the large differences between normal eyes and of those with retinal dystrophies in young subjects. The technique has the advantage of taking no more than 5 minutes to record the fluorescence of the fundus, and is non-invasive so that it could become widely used in clinical practice.

AvR was in receipt of grant no Ru 575/1-1 from the Deutsche Forschungsgemeinschaft, Bonn, Germany.

This study was supported by grants from The Medical This study was supported by grants from The Medical
Research Council, UK, and The American Retinitis Pigmentosa Foundation.

1 Remé C. Autophagy in visual cells and pigment epithelium. Invest Ophthalmol Vis Sci 1977; 16: 807-14.

2 Feeney L. Lipofuscin and melanin of human retinal pigment epithelium: fluorescence, enzyme cytochemical and ultrastructural studies. Invest Ophthalmol Vis Sci 1978; 17: 583-600.

3 Wing GL, Blanchard GC, Weiter JJ. The topography and age relationship of lipofuscin concentrations in the RPE. age relationship of lipofuscin concentrations

4 Eldred GE, Katz ML. Fluorophores of the retinal pigment epithelium: separation and spectral characteristics. Exp Eye Res 1988; 47: 71-86.

5 Weiter JJ, Delori FC, Wing GL, Fitch KA. Retinal pigment epithelial lipofuscin and melanin and choroidal melanin in human eyes. Invest Ophthalmol Vis Sci 1986; 27: 145-52.

6 Feeney-Burns L, Eldred GE. The fate of the phagosome: conversion to 'age-pigment' and impact in human retinal pigment epithelium. Trans Ophthalmol Soc UK 1984; 103: 416-21.

7 Eldred GE, Katz ML. Fluorophores of the retinal pigment epithelium. Exp Eye Res 1988; 47: 71-86.

8 Katz ML, Drea C, Robinson WG. Relationship between retinol and lipofuscin in the retinal pigment epithelium. Mech Ageing Dev 1986; 35: 291-305.

9 Rungger-Branche E, Englert U, Leuenberger PM. Exocytic clearing of degraded membrane material from pigment epithelial cells in frog retina. Invest Ophthalmol Vis Sci 1988; 28: 2026-37.

10 Dorey CK, Wu G, Ebenstein D, Garsd A, Weiter J. Cell loss in the ageing retina: relationship to lipofuscin accumulation and macular degeneration Invest Ophthalmol Vis Sci 1989; 30: 1691-9.

11 Wyszynski RE, Brunar WE, Cano DB, Morgan KM, Davis CB, Sternberg P. A donor age-dependent change in the activity of alpha-mannosidase in human cultured RPE cells. Invest Ophthalmol Vis Sci 1989; 30: 2341-7.

12 Boulton ME, McKechnie NM, Breda J, Bayly M, Marshall J. The formation of autofluorescent granules in 83-9.

13 Boulton M, Docchio F, Dayhaw-Barker P, Ramponi R, Cubbedu R. Age-related changes in the morphology, absorption and fluorescence of melanosomes and lipo- fuscin granules of the retinal pigment epithelium. Vis Res 1990; 30: 1291-303.

14 Boulton M. Aging of the retinal pigment epithelium. In Osborne NN, Chader GJ, eds. Progress in retinal research. Vol 11. Oxford: Pergamon Press, 1991: 125-52.

15 Weale RA. Do years or quanta age the retina? Photochem Photobiol 1989; 50: 429-38.

16 Hogan MJ. Role of the retinal pigment epithelium in macular disease. Trans Am Acad Otolaryngol Ophthalmol 1972; 76: 64-80.

17 Ishibashi $\mathrm{T}$, Sorgente $\mathrm{N}$, Patterson $\mathrm{R}$, Ryan SJ. Pathogenesis of drusen in the primate. Invest Ophthalmol Vis Sci 1986; 27: 184-93.

18 Sheraidah G, Steinmetz R, Maguire J, Pauleikhoff D, Marshall J, Bird A. Correlation between lipids extracted from Bruch's membrane and age. Ophthalmology 1993; 100: 47-51.

19 Holz FG, Sheriadah G, Pauleikhoff D, Bird AC. Analysis of lipid deposits extracted from macular and peripheral Bruch's membrane. Arch Ophthalmol 1994; 112: $402-6$.

20 O'Gorman S, Flaherty WA, Fishman GA, Berson EL. Histopathologic findings in Best's vitelliform macular dystrophy. Arch Ophthalmol 1988; 106: 1261-8.

21 McFarland CB. Heredodegeneration of macula lutea; study of clinical and pathological aspects. Arch Ophthalmol 1955; 53: 224-8.

22 Frangieh GT, Green R, Fine SL. A histopathological study of Best's macular dystrophy. Arch Ophthalmol 1982; 100: $1115-21$

23 Weingeist TA, Kobrin JL, Watzke RC. Histopathology of Best's macular dystrophy. Arch Ophthalmol 1982; 100: 1108-14.

24 Patrinely JR, Lewis RA, Font RL. Foveomacular vitelliform macular dystrophy, adult type. A clinicopathological study including electron microscopic observations. Ophthalmology 1985; 92: 1712-8.

25 Steinmetz RL, McGuire J, Garner A, Bird AC. Histopathology of incipient fundus flavimaculatus. Ophthalmology 1991; 98: 953-6.

26 Birnbach $C D$, Järveläinen $M$, Possin $\mathrm{DE}$, Milam $\mathrm{AH}$ Histopathology and immunocytochemistry of the neurosensory retina in fundus flavimaculatus. Ophthalmology 1994; 101 : $1211-9$.

27 Delori FC, Arend O, Staurenghi G, Goger D, Dorey CK, Weiter JJ. Lipofuscin and drusen fluorescence in aging and age related macular degeneration. Invest Ophthalmo Vis Sci 1994; 35 (suppl): 2145.

28 Elsner AE, Burns SA, Zenzie HH, Moulton PF. Infrared and autofluorescence imaging of subretinal structures. Optics Photonics News 1993; 4 (Suppl): 44

29 Fitzke FW, Woon H, Timberlake G, Robinson Marshall J, Bird AC. Optical modifications to a scanning laser ophthalmoscope for high magnification, narrow optical section imaging. Laser Light Ophthalmol 1991; 4: $7-14$.

30 Fitzke FW, Masters BR. Three-dimensional visualisation of confocal sections of in vivo human fundus and optic nerve. Curr Eye Res 1993; 11: 1015-8.

31 Kitagawa $K$, Nishida $S$, Ogura Y. In vivo quantitation of autofluorescence in human RPE. Ophthalmologica 1989 99: 116-21.

32 Wells J, Wroblewski J, Keen J, Inglehearn C, Jubb C, Eckstein A, et al. Mutations in the human retinal degeneration slow (RDS) gene can cause either retinitis pigmentosa or macular dystrophy. Nature Genetics 1993; 3: 213-7.

$33 \mathrm{Kim}$ RY, Dollfus H, Keen TJ, Fitzke FW, Arden GB, Bhattacharya SS, et al. Autosomal dominant pattern dystrophy of the retina associated with a 4 bp insertion at codon 140 in the RDS/peripherin gene. Arch Ophthalmo (in press).

$34 \mathrm{Kolb} \mathrm{H}$, Gouras P. Electron microscopic observations of human RP, dominantly inherited. Invest Ophthalmol Vi Sci 1974; 15: 487-98.

35 Flannery JG, Farber DB, Bird AC, Bok D. Degenerative changes in a retina affected with autosomal dominan retinitis pigmentosa. Invest Ophthalmol Vis Sci 1989; 30: 191-211.

36 Massof RW, Finkelstein D. Two forms of autosomal dominant primary retinitis pigmentosa. Doc Ophthalmol 1981; 51: $289-346$.

37 Arden GB, Carter RM, Hogg CR, Powell DJ, Ernst WJK, Clover GM. Rod and cone activity in patients with dominantly inherited retinitis pigmentosa: comparison between psychophysical and electroretinog
measurements. Br $\mathcal{F}$ Ophthalmol 1983; 67: 405-18.

38 Lyness AL, Ernst W, Quinlan MP, Clover GM, Arden GB Carter R. A clinical, psychophysical, and electroretinographic survey of patients with autosomal dominan retinitis pigmentosa. $\mathrm{Br} \quad \mathcal{f}$ Ophthalmol 1985; 69: 326-39.

39 Kemp CM, Jacobson SG, Faulkner DJ. Two types of visua disfunction in autosomal dominant retinitis pigmentosa. Invest Ophthalmol Vis Sci 1988; 29: 1235-41.

Note added in proof

Since submission of this manuscript the following publications have come to our attention: elori FC. Spectrophotometer for noninvasive measuremen of intrinsic fluorescence and reflectance of the ocula fundus. Applied Optics 1994; 33: 7439-52.

Delori FC, Dorey CK, Staurenghi G, Arend O, Goger DG Weiter JJ. In vivo fluorescence of the ocular fundus exhibits RPE lipofuscin characteristics. Invest Ophthalmol Vis Sci (in press). 